\title{
New spacecraft and protection of the planet from the asteroid hazard
}

\author{
Yuriy Alexeevich Baurov ${ }^{1,2}$, Lorenzo Albanese ${ }^{3}$, Francesco Meneguzzo ${ }^{3}$, \\ Valeriy Alexandrovich Menshikov ${ }^{4}$ \\ ${ }^{1}$ Closed Joint Stock Company Research Institute of Cosmic Physics, 141070, Moscow Region, Pionerskaya, 4, Korolyov, Russia \\ ${ }^{2}$ Hotwater Srl, , Via Gioberti, 15, I-56024 San Miniato (PI), Italy \\ ${ }^{3}$ National Research Council - Institute of Biometeorology, Via Caproni, 8, I-50145 Firenze, Italy \\ ${ }^{4}$ Non-Commercial Partnership (NP) "The International Committee of the Project of the International Global Aerospace Monitoring and \\ Forecasting” (IGMASS), 141092, Moscow region, Yubileynyy, Lesnaya, 14, Office 305, Russia
}

\section{Email address:}

baurov@mail.ru (Y. A. Baurov), 1.albanese@ibimet.cnr.it (L. Albanese), f.meneguzzo@ibimet.cnr.it (F. Meneguzzo), vamenshikov@mail.ru (V. A. Menshikov)

\section{To cite this article:}

Yuriy Alexeevich. Baurov, Lorenzo Albanese, Francesco Meneguzzo, Valeriy Alexandrovich Menshikov. New Spacecraft and Protection of the Planet from the Asteroid Hazard. American Journal of Astronomy and Astrophysics. Special Issue: Global Anisotropy, Theory of Byuon, New Force, New Power System, Propulsion, Space Flights. Vol. 2, No. 6-1, 2014, pp. 54-61. doi: 10.11648/j.ajaa.s.2014020601.18

\begin{abstract}
A project is presented to create in Mars' orbit a protective stations belt (PSB) designed to bring down the asteroid hazard to the Earth, based on an interplanetary spacecraft(ISC) of a new type. This project may as well contribute to the investigation of fundamental problems of astronomy and astrophysics in the study of the properties of deep space using space platforms. The project is based on technical realizations which feasibility derives from the cosmological theory of byuon (TB) about the formation of the physical space and the world of elementary particles along with the experimental findings about new properties of nature: a new force that is used in the form of thrust for spacecrafts and a new quantum information channel providing a connection between the Earth, the PSB and the ISC. The results of experimental investigations about the traction force with a ground model of spacecraft are recalled, confirming the existence of a global anisotropy of physical space as predicted by the theory of byuon, as well as the technical feasibility to harness the new force in order to implement new concept space thrusters significantly more energy-effective than existing models. An action plan to implement the creation of the ISC is worked out.
\end{abstract}

Keywords: Global Anisotropy, Theory of Byuon, Traction Force, Propulsion, Space Flights, Asteroid Hazard

\section{Introduction}

This article describes the implementation of the interplanetary project developed by the Research Institute of Space Physics (Russia), NP IGMASS (Russia) and Hotwater Ltd, (Italy) to protect the planet Earth from the asteroid hazard. The extent of this danger exceeds by far that of any other natural disasters (earthquakes, tsunamis, tornadoes, etc.) as well as of technological disasters because as a consequence of the impact of a medium-size asteroid (around $10 \mathrm{~km}$ in size) any or most of life on planet Earth could cease.

Fig. 1 shows the inner solar system (from the Sun to Jupiter), including the asteroid belt (ring-shaped white cloud) and other groups of asteroids.

This area is often called the main asteroid belt [1] or just the main belt [2], thus emphasizing its difference from other similar areas with clusters of small planets, such as the Kuiper Belt beyond Neptune's orbit, as well as with clusters of objects scattered in the disc or the Oort cloud.

The total mass of the main belt is about $4 \%$ of the mass of the Moon, more than half of it being concentrated in the four largest objects: Ceres, Pallas, Vesta and Hygeia. Their average diameter is more than $400 \mathrm{~km}$, and the largest of them, Ceres, the only dwarf planet in the main belt, has a diameter larger than $950 \mathrm{~km}$ and twice the total mass of Pallas and Vesta. Yet, the majority of asteroids, in the number of several millions, have considerably smaller diameters, up to several tens of meters. Nevertheless, the probability of asteroids colliding among themselves and with comets flying through this belt is not zero, as well as the Earth itself could collide with a medium-sized asteroid, even one that has not been previously observed from the Earth. On our planet, 
there are imprints of events of this magnitude, such as in the Gulf of Mexico basin.

A series of events since the early XX century (Tunguska meteorite, Sikhote-Alinsky meteorite with known mass about 28 ton and total mass up to 100 ton, etc.) shows that the asteroid hazard increases due to increased urban sprawl and the growth of settlements. The latter event, associated with Chebarkulsky meteorite in 2013, could well have resulted into a huge catastrophe for the city of Chelyabinsk, Russia, avoided just by chance.

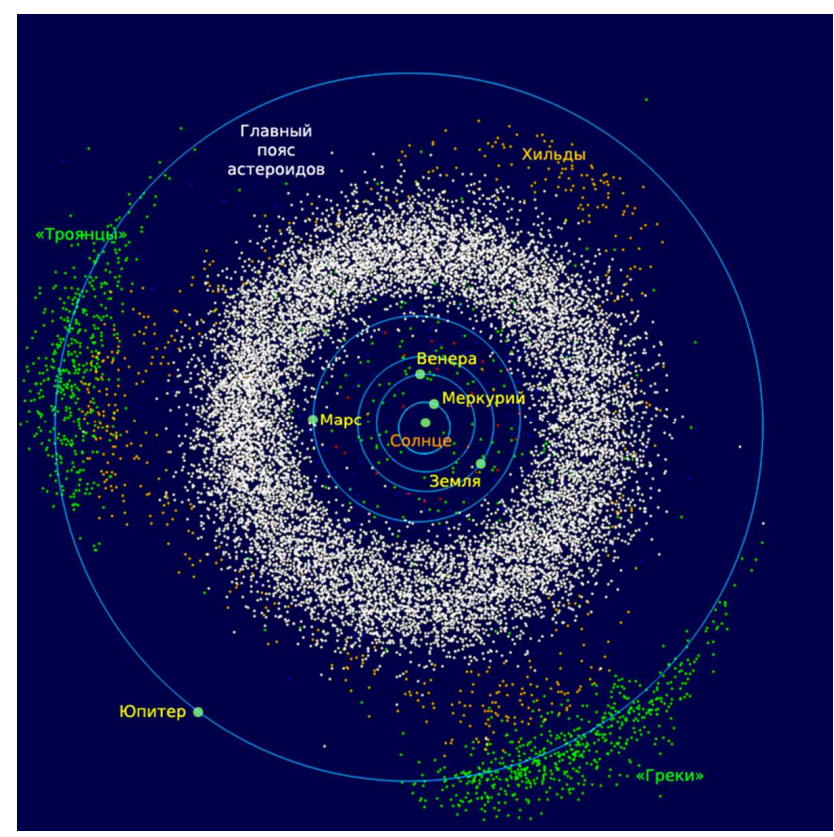

Fig. 1. The inner solar system (from the Sun to Jupiter), including the asteroid belt (white cloud of annular shape), a group of asteroids called "Hilda" (orange "triangle" just inside the orbit of Jupiter) and Jupiter Trojans (green). The group, which is ahead of Jupiter, called "Greeks", and the closing group called "Trojans". Image based on the ephemeris data DE405 from Jet Propulsion Laboratory (JPL) and database of asteroids from Minor Planet Center (MPC) at the Smithsonian Astrophysical Observatory, published on July 6, 2006. The picture represents the situation on August 14, 2006 , with the view from above on the plane of the ecliptic.

Substantial danger to the Earth could be created during this century by the asteroid Apophis (mass around $4.6 \cdot 10^{10} \mathrm{~kg}$, entry speed in the atmosphere of $12.6 \mathrm{~km} / \mathrm{s}$, characteristic size of $325 \pm 15 \mathrm{~m}$ ) [3]. The initial assessment by NASA for the equivalent of TNT explosion in the fall of the asteroid was 1480 megatons (Mton) and later, after verification of sizes, it was reduced to 880 Mton and then further down to 506 Mton. For comparison, the energy release at the impact of the Tunguska meteorite is estimated to have been 10-40 Mton.

Following the radar observations, the possibility of collision with Apophis in 2029 was excluded, but due to inaccuracies in the initial data the probability of collision of this object with our planet exists in 2036 and subsequent years; various researchers evaluated the mathematical probability of collision between $2.2 \cdot 10^{-5}$ and $2.5 \cdot 10^{-5}$.

The effect of explosions resulting from the impact of Apophis may vary depending upon the composition of the asteroid, as well as the location and angle of incidence. In any case, the explosion would cause enormous damage on an area of thousands of square kilometers but will neither destroy the planet nor would create long-term global effects such as an "asteroid winter." In any case, even the impact of a minor asteroid like Apophis can lead to consequences that couldn't likely be generated by any of the other classes of natural disasters, therefore the asteroid hazard can be considered the primary source of danger for mankind.

In this paper, we point out that using standard physics and existing technologies to solve the problem of protecting the planet from the asteroid threat is practically impossible. We'll show as well that there is a new physical model describing the world around us, the theory of byuon (TB) [4-7], nongauge theory of the formation of the physical space and the world of elementary particles from non-observable objects called byuons, which predicts the existence of a new interaction in nature besides the known ones, i.e. strong, weak, electromagnetic and gravitational, as well as a new quantum information channel (QIC) linking together all the objects of nature into one common information field. The physical reality of both the new force of nature and the QIC were experimentally confirmed in recent years [8-10].

Using these two new physical effects, i.e. the new force of nature in the form of thrust for spacecrafts, and the QIC to transmit quantum information in outer space, the project of creation of a protective stations belt (PSB) that shields the planet from the asteroid hazard becomes feasible.

\section{Protective Stations Belt and Interplanetary Spacecraft of a New Type to Prevent Asteroid Hazard}

The creation of the PSB dictates the following stringent requirements for the implementation of anti-asteroid hazard in the following areas:

- Geometry of the arrangement of the PSB;

- Creation of an international spacecraft (ISC) that can quickly reach high enough speeds ensuring the interception of asteroids and their destruction or transfer to a safe orbit;

- Creation of communication between PSB, Earth and ISC, providing the solution to the problem.

In order to secure the planet from the asteroid hazard within these requirements it is proposed to arrange 6 monitoring stations on the orbit of Mars on their own platforms, along with the ISC of a new type, which will be able to reach the speed necessary to intercept the asteroid. The orbital location of the PSB is clear from consideration of Fig. 1 as the main asteroid belt is located between Mars and Jupiter. The number of stations is determined by the thrust-toweight ratio of the ISC, that must solve the problem of reaching the asteroid before a limiting distance to the Earth, resulting in its safety.

If PSB will have six stations, the average distance to intercept at different angles of approach of the asteroid will 
be near 1 a.u. (astronomical units) and the ISC, having a thrust-to-weight ratio at the level of $(1 / 6) \cdot g$, where $g$ is the acceleration of gravity on the Earth, can intercept in a time about $3 \cdot 10^{6} \mathrm{~s}$; such time is shorter than the time required for asteroids in the main belt, moving at the maximum observed speed of about $30 \mathrm{~km} / \mathrm{s}$, to reach the Earth. Let's note that the creation of such ISC will allow to begin the expansion of humanity into the outer space: the creation of settlements on Mars (the first and primary of the six stations will be placed on this planet) and the Moon and, with the above specified thrust-to-weight ratio, such a spacecraft will be able to land and take off from the Moon by itself, without using a landing module.

On the Moon it's also assumed to create a station (or system of stations) with information purposes, aimed at the observation of asteroids and other space objects, as well as to address the fundamental problems of the universe; due to its location, of course such station cannot replace the PSB and accomplish its tasks.

Unfortunately, the currently existing technology and standard physical models can't solve the technical problem of creating the PSB: solution to this problem can only be based on new physical principles.

Let us dwell on the physical principles of implementation of a new type of ISC and its design.

For many years in Russia in the Laboratory for perspective energy systems at TsNIIMash (Russia, Korolev) a new energy source has been investigated, such source being associated with the opportunities to affect the formation of masses of elementary particles using potentials of physical fields as unveiled within the framework of the TB.

According to the theory of byuon a fraction of the mass of the elementary particles, associated with the formation of their inner physical space and up to the value $\Delta \mathrm{m} \cdot \mathrm{c}^{2}=33 \mathrm{eV}$ in value, is proportional to the modulus of a summary potential $A_{\Sigma}$, i.e. the sum of the potentials of all known force fields calculated using the energy relation expressed by Eq. (3.1.) in [11]. Such summary potential cannot exceed, by magnitude, the modulus of the cosmological vectorial potential $\mathrm{A}_{\mathrm{g}}$, a new fundamental constant having absolute value $\left|\mathrm{A}_{\mathrm{g}}\right| \approx 1.9 \cdot 10^{5} \mathrm{~T} \cdot \mathrm{m}$. As the result of the action of the field potentials (decreasing $\left|\mathrm{A}_{\Sigma}\right|$ ), each particle gains an energy $\Delta \mathrm{m} \cdot \mathrm{c}^{2}$, c being the speed of light, that corresponds to a new force of nature throwing substance out of the region with the weakened $\mathrm{A}_{\Sigma}$.

The interested reader is invited to get acquainted with the foundations, features and experimental confirmations of the $\mathrm{TB}$ as described in several recent articles, such as $[7,12,13]$, as well as with the unambiguous detection of the predicted new force of nature along with its practical use in the form of traction $[10,14]$.

Companies Hotwater (Italy) and JSC Research Institute of Cosmic Physics (Russia) have been undertaking work to harness the new force into new concept thrusters for spacecrafts, while the first experimental studies in this direction were conducted in TsNIIMash where the earliest experimental models of thrusters using a new force in the form of thrust were created [15]. With a mass of the model of
$4.5 \mathrm{~kg}$ and using a strain-gauge balance, a traction about $10 \mathrm{~g}$ was detected during 1-2 minutes, while the specific power, as a measure of the thrust efficiency, was at the level of $0.03 \mathrm{~W} / \mathrm{g}$, i.e. about 4,000 times better than the most effective electric propulsion (EP) engines and in particular of Hall effect thrusters (HETs) [16], which are currently considered the most promising for the realization of a flight to Mars $[17,18]$.

Nevertheless, neither sustainable mechanisms for traction control nor sustained traction for a sufficiently long time could be obtained at TsNIIMash [14]; such results were obtained for the first time in Italy in 2012 -2013 [10].

The first working model demonstrating a sustained traction in time and thus the possibility of its practical use for space applications was created and is currently operated in Italy, using the new force of nature in the form of traction: the "universal thruster", schematically shown in Fig. 2, is placed onboard a polycarbonate hemispherical ship model with deviation from sphericity at the level of $0.1 \%$, minimizing any systematic errors, the effects of inertia, as well as differential friction forces in opposite directions. The results of a long experimental run during the period November, 2012 to September, 2013 are comprehensively described in [10], as well as the first phase of the same experimental run is partially summarized in a short movie [19].

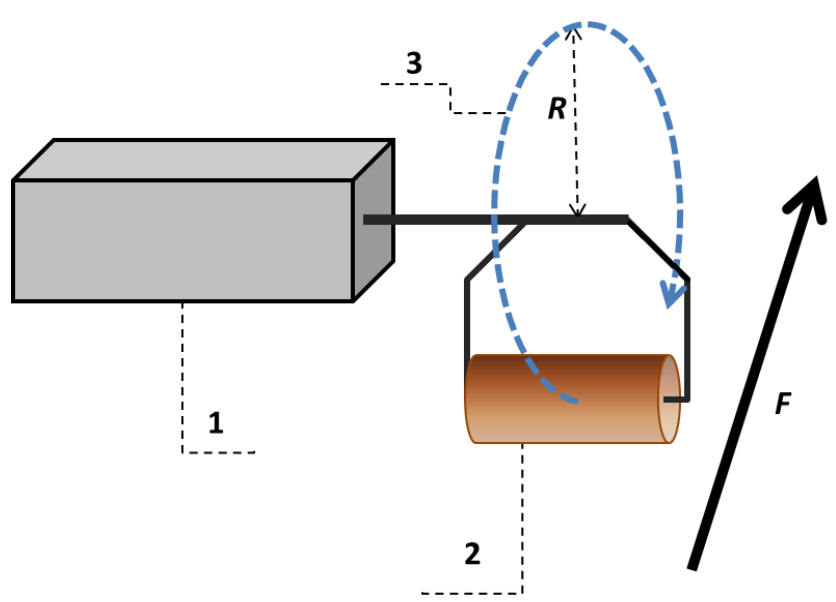

Fig. 2. Schematic view of the new thruster. 1 - electromotor (i.e. a step motor); 2 - off-center iron load; 3 - rotational trajectory of the load, periodically resting along or near the vertical; $R$ - revolution radius, not less than $5 \mathrm{~cm} ; \mathrm{F}$ - expected vector of action of the new force, producing cyclic impulses just before each stop of the load, opposite to the direction of motion of the load itself.

The peak traction force was obtained on January 27, 2013, at the value of about $50 \mathrm{~g}$ corresponding to the off-center load performing a cycle with a period of revolution equal to $0.115 \mathrm{~s}$ and stopping in the lowest position during $0.12 \mathrm{~s}$; the traction force was generally 5 to 10 times greater than the measurement error, while the lowest specific power was around $3.3 \mathrm{~W} / \mathrm{g}$, i.e. about forty times smaller than the corresponding benchmark figure of the most efficient HETs used in the space technology for the propulsion of satellites and spacecrafts.

It's remarkable that the experimental results are in 
qualitative agreement with the theoretical estimate of the range of the new force.

The analysis of a long run of experiments $[4,5]$ has shown that the new force has a nonlinear and nonlocal character and can be represented as a complex series in terms of changes of the summary potential $A_{\Sigma}$. The first term of the series is the following:

$$
\mathrm{F}=2 \mathrm{Nm}_{\mathrm{v}} \mathrm{c}^{2} \lambda_{1}^{2} \Delta \mathrm{A}_{\Sigma}\left[\Delta\left(\Delta \mathrm{A}_{\Sigma}\right) / \Delta \mathrm{x}\right]
$$

where $\mathrm{N}$ is the number of stable particles (electrons, protons, and neutrons) in the test body, $\Delta \mathrm{A}_{\Sigma}$ is the difference in changes of the summary potential $A_{\Sigma}$ at the location points of a test body and sensor element, $\Delta\left(\Delta \mathrm{A}_{\Sigma}\right) / \Delta \mathrm{x}$ is the gradient in space of the difference potentials $\Delta \mathrm{A}_{\Sigma}$; $\mathrm{x}$ is the length of an arc of a circle for experiments with solenoids, therefore a space coordinate; $2 \mathrm{Nm}_{\mathrm{v}} \mathrm{c}^{2}=33 \mathrm{eV} ; \lambda_{1}=10^{-6}(\mathrm{Tm})^{-1}$ is the first coefficient of the series.

The upper value of the traction force can be estimated replacing the quantities in (1) with the appropriate numbers; given the mass equal to $526 \mathrm{~g}$ and material, i.e. iron, of the off-center load of revolution we have: $\mathrm{N} \approx 10^{26}$ and, according to [4,5,12]: $\Delta \mathrm{A}_{\Sigma}=\Delta\left(\Delta \mathrm{A}_{\Sigma}\right), \Delta \mathrm{A}_{\Sigma}=\mathrm{A}_{\mathrm{g}} \cdot \cos _{\text {II }}^{++} \cdot \mathrm{c} / \mathrm{v}, \cos _{\text {II I }}^{++}=10^{-15}$, where $\mathrm{c}$ is the speed of light, $\mathrm{v}=\Delta \mathrm{x} / \Delta \mathrm{t}=0.02 \mathrm{~m} / \mathrm{s}$ is the extinction velocity of the Information Object (IO) of the mentioned load, where $\Delta \mathrm{x}$ is the characteristic size of the body of revolution, i.e. around $2 \mathrm{~cm}$ in the performed experiments, and $\Delta \mathrm{t}$ is the extinction time of the IO, on the order of $1 \mathrm{~s}$ as stated in[10]. Substituting into (1), the upper limit of the traction force can therefore be estimated on the order of $1 \mathrm{~N}$.

During the experiments in Italy the global anisotropy of the physical space associated with the cosmological vector potential $A_{g}$ was further confirmed: in the period February 6 to August 6, 2013, the intensity of the new force weakened by about 5 times or more with regards to other half of the year. Within an accuracy on the order of $10^{\circ}$, the line drawn from a point on the Earth's orbit corresponding to August 6 to the point corresponding to February 6 coincides in direction with the coordinate of right ascension obtained in recent studies for the vector $A_{g}$, at the value of about $300^{\circ}$ [7].

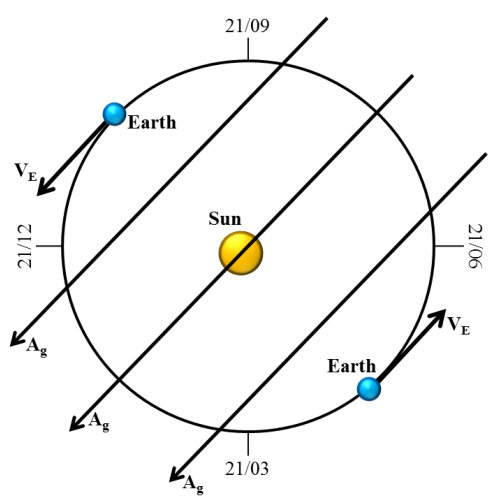

Fig. 3. Projection of the cosmological vector potential $A_{g}$ onto a simplified Earth's orbital plane; $V_{E}$ is the Earth's instantaneous velocity vector.

Fig. 3 shows the direction of the cosmological vector potential $A_{g}$ projected onto the Earth's orbital plane, representing the direction of the global anisotropy of the physical space.

For the new proposed ISC no fuel or working fluid is required onboard, therefore its operating time is limited only by the availability of power needed to rotate the loads according to special cycles, the basic idea of the new propeller being protected by a patent [20]. Since the reduced specific power of the thruster allows the generation of all the primary energy needed by the spacecraft by means of solar panels, its operating time in space while in the solar neighborhood will be practically unlimited, thereby reducing the operational requirements to perform its main task, i.e. changing a dangerous orbit of an asteroid to a safe one, to get a constant thrust of about $50 \mathrm{t}\left(5 \cdot 10^{3} \mathrm{~kg}\right)$.

Let's note that in addition to solving the basic problem, the PSB will increase the scope of observational astronomy in the exploration of dark matter, antimatter, the structure of the nuclei of galaxies and so on. In connection with the anticipated increase in the base length up to the size of the orbit of Mars, the sensitivity of radio astronomy will increase by orders of magnitude because its resolving power depends on the ratio of the wavelength to the length of the measurement base.

Let's note that the elements of the observation base (platforms of PSB) will be linked together by means of a quantum information channel (see Section 4. below and $[4,8,21]$ ) transmitting information without delay (practically, any delay will be caused only by electronic devices implementing the new physical method of transmitting information).

\section{Bases of Design of the Interplanetary Spacecraft}

The principle of design of the described ISC is proposed in a patent [22]. The sizes of the ISC for the values of the specified traction (50 t) are shown in Fig. 4 and Fig. 5 (solar panels not shown).

The ISC is planned to be assembled in orbit, using the International Space Station (ISS) as a base for the constructors; during the construction process, the gravity shall be used for the stabilization of the complex object ISSISC.

Furthermore, on the basis of the new force of nature, special "space scooters" will be created that will allow astronauts to move freely enough in outer space and combine in a uniform construction the elements of the ISC while it's normally in orbit around the Earth.

Currently, a framework of technical proposals has been developed for the creation of the ISC.A distinctive feature of the design of this spacecraft is the fact that this unit does not use the reaction (jet) principle of motion, rather harnessing the new force of nature to receive the needed thrust. The ISC will use as the reference environment the physical space as a quantum medium (dark matter) with which it will exchange 
momentum in the course of the flight $[4,6,10,14]$.

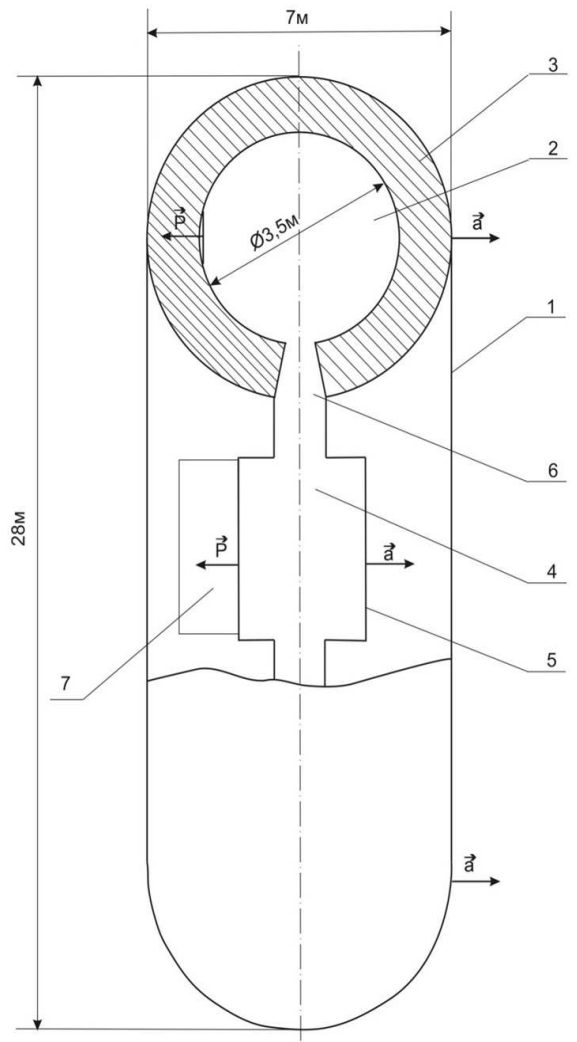

Fig. 4. ISC Section of toroidal shape for the flight to Mars, using the new energy : 1 - ISC toroidal body; 2 - working section and toroidal shape housing; 3 - ISC traction compartment; 4 - monitoring cabin with porthole (5); 6 - passage compartments; 7 - compartment of the landing module; $\vec{P}-$ direction of the artificial gravity; $\vec{a}$ - direction of the ISC acceleration.

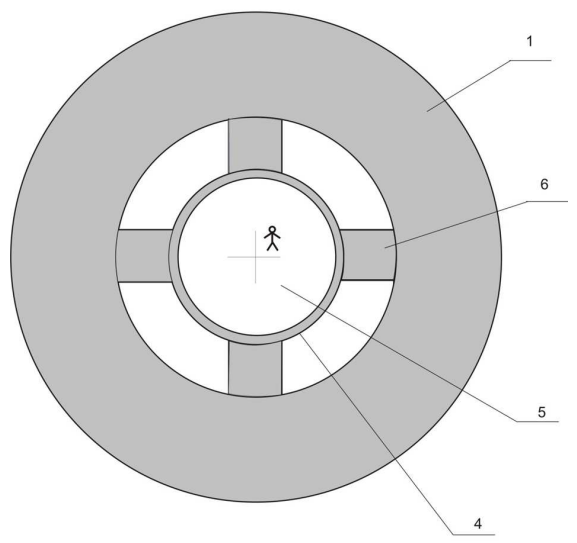

Fig. 5. General view of the ISC for the flight to Mars, using the new energy, scale 1:200. 1 - ISC toroidal body; 4 - monitoring cabin with transparent porthole (5);6 - passage compartments.

Preliminary calculations show that with the size of the toroidal ISC shown in Fig. 4 and Fig. 5 and the weight of the whole construction around $300 \mathrm{t}$, it's possible to supply traction at the level of $50 \mathrm{t}$ using 24 traction sections, each of which producing a thrust greater than $2 \mathrm{t}$, possessing 12 unit thrusters each of which in turn generating $200 \mathrm{~kg}$ of traction, allowing the ISC to land on the Moon without the use of other types of engines. The principle of construction of the traction sections is based on the design of the ship described in [22], and with the characteristics of the rotating bodies (Fig. 2) obtained in the above mentioned experiments carried out in Italy in 2012-2013 [10].

The planned traction $(50 \mathrm{t})$ will deliver an expedition to Mars and bring the crew back in one month, staying on Mars during 10 days. It is clear that such reduced mission time as well as the construction described above for the ISC, will help keeping all risks connected with planetary expeditions to a minimum, in example the effects of radiation from solar flares, possible loss of consciousness of the crew for a long flight [23], technical failures, etc. Let's note further that the ISC will always move with acceleration around (1/6).g, significantly reducing the effect of weightlessness (reduced gravity) on the crew.

In order to confirm the above, let's compare the proposed characteristics with the project characteristics of a spacecraft designed for a flight to Mars using, in example, nuclear power propulsion units (NPPUs), various versions of which are presented in [18].

With a bimodal type of installation (NPPU both powering the propulsion unit and generating electricity) with turbomachine energy conversion and a complex of four modules, NPPUs create traction at the level of $68 \mathrm{kN}(6.8 \mathrm{t})$. The resulting thrust ensures a time of expedition to Mars equal to 460 days. With the use of electric propulsion (EP) the time of expedition could be reduced to 328 days (with 1 month on Mars) [18]. Therefore, even the use of EP cannot reduce the mission time below an order of magnitude greater than using the new force of nature in the form of thrust for the spacecraft, and their typical specific power figures around $130 \mathrm{~W} / \mathrm{g}$ are more than one order of magnitude greater than the one offered by the new proposed type of propulsion. It should be noted as well that for the creation of an NPPUs complex for a flight to Mars it's necessary to address a series of complex technical problems such as the development of structures operating at temperatures ranging from that of liquid hydrogen to $3,000 \mathrm{~K}$, the issues of heat dumping, etc.

Several details contribute to enhance the problems connected with heat dumping in a spacecraft powered by onboard NPPUs, starting from the consideration that the spacecraft in the cosmic vacuum is basically a thermally insulated body, and creating a cooler radiator to dump the excess heat is a complex problem when using NPPUs. If on Earth as a result of an accidental burst of nuclear activity the problem can be solved quickly by removing the heat by means of the cooling circuits, in space a quick addressing of this problem is impossible. In [24,25] it is shown that in nature can be observed changes in the rate of $\beta$-decay of radioactive elements from $1 \%$ to $1000 \%$ above the background value, moreover events in which the decay rate increases up to $1000 \%$ can be observed up to 4 times throughout the year [25]. Should such an event occur during a flight to Mars using the NPPUs, the mission itself would be threatened with destruction. Therefore, the use of NPPUs for an expedition to Mars would carry along a significant and intrinsic risk. 


\section{The New Quantum Information Channel}

In order to implement the program of creation of the PSB, the existing means of communication on the basis of electromagnetic waves just aren't sufficient. In example, the conventional electromagnetic signal from Earth to Mars takes $3 \mathrm{~min}$ to $20 \mathrm{~min}$, depending on the relative positions of the planets. During this time, the parameters of approach to an asteroid can change dramatically, the decision chain could suffer from a fatal delay and the task of intercepting the asteroid would be missed.

In addition to a new force of nature, the TB predicts the existence of the QIC, linking all objects of nature into one common information field. The latter is due to the fact that the object of this theory, i.e. a four contact interaction byuon ("object $4 \mathrm{~b}$ "), has an interval of uncertainty of Heisenberg in the spatial coordinate equal to the size of the observable Universe, i.e. $10^{28} \mathrm{~cm}$. This object defines the observed density of matter in the universe, around $10^{-29} \mathrm{~g} / \mathrm{cm}^{3}$ and reveals the physical nature of dark matter $[5,6,12]$. Note that in the TB there is no speed of objects exceeding the speed of light, and the postulate of the special relativity theory on maximum speed of objects equal to the speed of light derives from the postulates of TB. The new communication channel also defines the origin of life in the universe, because all living objects use this channel to suppress the growth of entropy $[9,21]$, that otherwise would lead to the death of any complex system. In particular, in [4,9] is shown that the nature of human consciousness is based on the existence of QIC, as at the typical scale of $10 \mathrm{~cm}$ of our brain, its biological objects can exchange information without delay, just using the QIC. The latter is based on the fact that, for example, an electron shares complete information associated with another electron at a distance of $10 \mathrm{~cm}$ on the basis of the QIC. The latter leads to one important feature for the crews of a spacecraft which can for long time (more than one month) miss out the properties of the physical space, the birthplace of their consciousness, such as the influence of the magnetic potential, the gravitational fields of the Earth and its other properties affecting the object $4 \mathrm{~b}$. The crew may lose consciousness while away from the Earth's magnetosphere for a long time [23], just because of the change in the object $4 \mathrm{~b}$. In this respect, the new spacecrafts should include in their living area a "corner" of our planet with its basic properties, in particular, onboard the spacecraft there should be a magnetic system generating a magnetic field on the order of the terrestrial one $(0.4 \mathrm{G})$.

In $[4,5,8,21]$ the existence of signals of a new nature is experimentally confirmed as well as a receiving device of these signals passing through the QIC is designed on the basis of systems of crystal oscillators. A method to receive a new type of information is described below, as the basis to plan the space communication infrastructure based on QIC.

The sensitive element of the experimental stand designed for the investigation of the QIC is the quartz resonator of the quartz generator QG1 placed within the magnetic system of permanent magnets creating a magnetic field of about $3500 \mathrm{G}$ in the magnetic circuit. The quartz resonator is mounted on a conducting base that allows it to be positioned in the space region with the minimal field of $\mathrm{B} \approx 1 \mathrm{G}$. The magnetic system is the source of the vector potential field and of the gradient of the change in the modulus of the summary potential $\mathrm{A} \Sigma$ (i.e., $\partial \Delta \mathrm{A} \Sigma / \partial \mathrm{x}$, see eq. (1)) that comprises the above-mentioned cosmological vector potential $\mathbf{A}_{\mathbf{g}}$ and the potentials of all natural and artificial magnetic sources.

The magnet system acts as an amplifier of signals passing through the structure of physical vacuum. Such signals, i.e. any changes in $\Delta \mathrm{A}_{\Sigma}\left(\partial \Delta \mathrm{A}_{\Sigma} / \partial \mathrm{x}\right)$, by themselves may have negligibly small amplitudes because of the immense cosmic scale of the regions of $A_{\Sigma}$ changes and, therefore, may be practically unobservable without the magnetic system.

The system registering the new signals includes a reference quartz resonator of generator QG2 situated outside the magnetic system, about $1 \mathrm{~m}$ away from QG1. Both quartz resonators and the electronic unit are housed in a closed thick-wall metal chamber, which significantly screens the external electromagnetic interference and also acts as a thermal damper and shield against various extraneous influences (such as air convection). Beside the chamber is a personal computer registering the experimental parameters.

Fig. 6 show a block diagram of the electronic devices for monitoring the operation of the system. In order to obtain precise measurements of the changes in the frequency $\mathrm{f} 1$ of the QG1 quartz resonator, as indicated above, the reference generator QG2 with frequency $\mathrm{f} 2$ is used. The measured frequency difference $\Delta \mathrm{f}=\mathrm{f} 2-\mathrm{f} 1$ between $\mathrm{QG} 2$ and QG1 characterizes, in the framework of our concept, the change in $\mathrm{A}_{\Sigma}$ and hence the value of the new force. When the phase shift between the QG2 and QG1 oscillations reaches $\Delta \varphi=$ $360^{\circ}$, the synchronous phase detector unit generates a pulse.

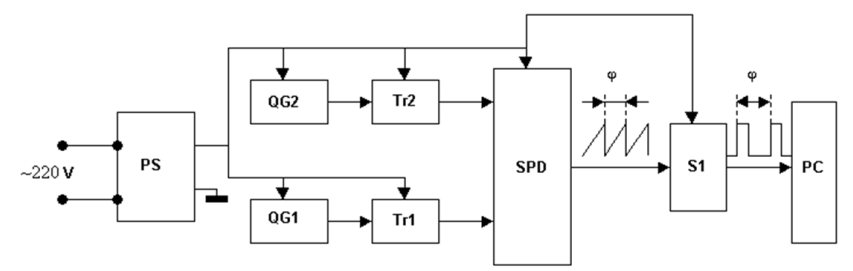

Fig. 6. Block diagram of the electronic devices. PS: power supply (stabilized voltage); QG1: quartz generator with the resonator placed within the vector potential field of permanent magnets; QG2: reference quartz generator; $\operatorname{Tr} 2$ and Tr1: triggers, scale-of-two frequency dividers producing a meander signal to the output pulses of quartz generators with frequencies $f_{2}$ and $f_{1}$; SDP: synchronous phase detector determining the angle of shift of the QG1 frequency relative to the reference one i.e. QG2; S1: shaper of standard output pulses for the PC with the pulse fall corresponding to the moment of time when the phase difference between the QG2 and QG1 oscillations reaches $\Delta \varphi=360^{\circ}$.

The sequence of pulses produced by the electronic scheme is fed to the PC where the time intervals between the pulse trains are determined to an accuracy of about $5 \cdot 10^{-7} \mathrm{~s}$. To ensure such accuracy, the measuring board has a quartz resonator with a frequency of $10 \mathrm{MHz}$ that sets a continuous sequence of time strobes with duration $10^{-7} \mathrm{~s}$. 
The results of measurements and the current time are displayed at the PC terminal and simultaneously recorded on hard disk for storage and further processing.

During the experiment, the influence of various external factors (temperature, pressure, etc.) was investigated [5]; for example, let's consider the effect of temperature during few days.

To check this issue, frequent measurements of temperature during almost three days were taken [5,8]. Fig. 7 shows the simultaneous series of the phase difference of the quartz resonators and of the temperature.

It arises from Fig. 7 that the temperature series does not correlate with the series of the basic signal and therefore cannot be used for explanation of the main result obtained, i.e. the intraday change of phase difference of two quartz generators. In this experiment, the main signal reflects the change of the summary potential due to the vector potential of the Earth's magnetic field.

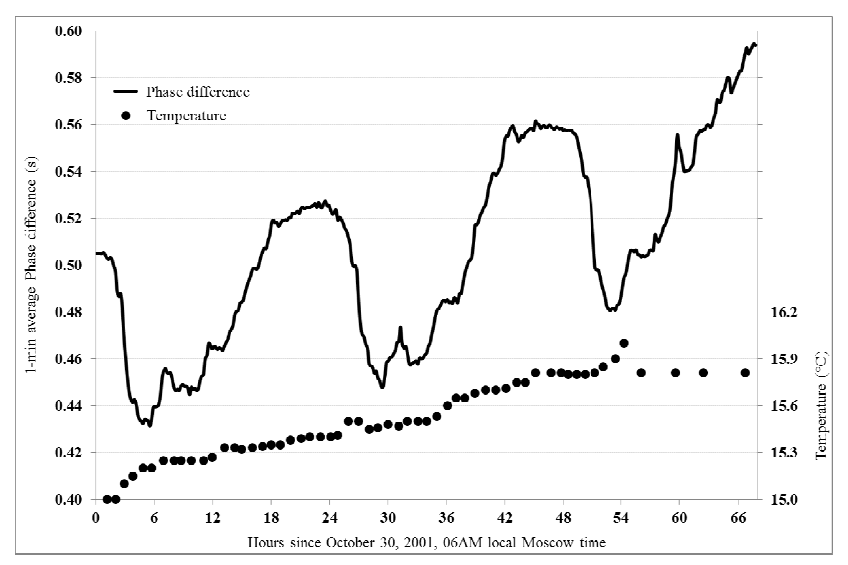

Fig. 7. Phase difference of the quartz resonators and temperature time series during about 68 hours starting on October 30, 2001, O6AM local Moscow time.

In [5] are shown other experiments to investigate the QIC (such as experiments with a high-accuracy quartz gravimeter "Sodin" and observation of changes in the rate of $\beta$-decay of radioactive elements), but the authors think that the connection between PSB, ISC and Earth can be practical only if built on the basis of a system of ideal crystals having the same values of the coefficients $\lambda_{i}(i=1,2,3$,.) belonging to the series describing the new force $[4,5]$. With exactly the same values of these coefficients it will be possible to transmit the information about any event almost instantaneously.

\section{International Cooperation, Funding for the Project and Time of Implementation}

Currently in the world (USA, etc.) exists and is developing a social movement to establish a fund for the development of our civilization and the colonization of the planet Mars as well as for the Moon exploration, with financial figures around US\$ 150 billion. The proposed technical solution for the project implementation of ISC allows a decisive simplification of the construction of spacecraft, a reduction of the project cost by one order of magnitude to implement the bulk of the project of the creation of the ISC, with ground tests of power units, control systems, etc., funded by Governments and private capital too.

It is assumed that the campaign can be carried out for all countries of the world, but mainly in countries participating to the ISS community: U.S.A., Russian Federation, Europe, Japan, as well as China and India.

In the development of the project it's is planned - even before the start of the flight to the Moon and Mars - to pay back a significant part of the cost of the expedition as result of the sale of effective, environmentally friendly, ground installations using the new force of nature to produce energy.

Due to the significantly simpler design of the ISC compared to all analogous existing projects, and the fact that the main design features to implement the ISC have already been developed, the first phase of PSB, i.e. development and creation of the ISC with its first landing on the Moon and the flight to Mars, can be completed in $7-8.5$ years [26]:

- Development of conceptual design: 1 - 1.5 years;

- Creation of basic blocks of ISC, creation of stands for the respective testing and in particular for the measurement of the tractive forces and test of control system, etc.: $2-2.5$ years;

- Assembling the ISC on the Earth and conducting trial device tests of the ISC on a special integrated stand: 1 1.5 years;

- Creation on the ISS of a berthing platform at a distance of about $100 \mathrm{~m}$ from the ISS (towards the Earth) for the installation of the ISC: same period of time as the previous item;

- Disassembly of the ISC and placement of units into orbit from subsequent installation of the ISC on the basis of ISS: 2 years;

- Trials of the ISC in Earth orbit, and the first flight to the Moon: 0.5 years;

- Preparing and conducting a test flight to Mars with a flight around the planet: 0.5 years.

\section{Conclusions}

The proposed project of creation of the PSB designed for the protection of the Earth from the asteroid hazard is based on recently unveiled physical phenomena: a new force of nature and a new quantum information channel, studied experimentally by different groups of experimenters on the best experimental bases of Russia for more than 20 years and, in recent years, in Italy; the physical basis of the project can be considered well established.

The proposed new principle of motion for the ISC and its design are protected by patents and are completely original. These allow to implement the PSB on the basis of an international cooperation among interested States and organizations in the course of the foreseeable future, till the implementation of the first flights of the ISC to the Moon and to Mars. 
The creation of the ISC using the ISS as a construction base is deemed to be possible within $7-8.5$ years.

\section{Acknowledgments}

The authors gratefully acknowledge C. Dionisio for valuable discussions about spacecraft propulsion and operations; M. Pagliaro for the outstanding support and motivation by means of the SuNEC 2013 Conference; F. Zabini for assistance in the communication style of the manuscript.

\section{References}

[1] G. Ranzini, Atlas de l'univers, Proxima Editions, Paris, France(in French), 2002.

[2] E. V. Kononovich, V. I. Moroz, General Course of Astronomy (in Russian), Editorial URSS, Moscow, Russian Federation, 2004.

[3] L. L. Sokolov, A. A. Bashakov, N. P.Pit'ev, "Specific Features of Asteroid 99942 Apophis Motion", Astron. Vestn., 42(1), pp. 20-29, 2008.

[4] Yu. A. Baurov, On the structure of physical vacuum and a new interaction in Nature (Theory, Experiment and Applications), Nova Science, NY, U.S.A., 2000.

[5] Yu. A. Baurov, Global Anisotropy of Physical Space, Experimental and Theoretical Basis, Nova Science, NY, U.S.A., 2004.

[6] Yu.A. Baurov, Yu.A., La trama svelata. La Teoria Byuon: dall'origine dell'Universo alla tecnologia del futuro, passando per l'Uomo, Polistampa, Firenze, Italy (in Italian), 2009.

[7] Yu. A. Baurov, "Research of global anisotropy of physical space based on investigation of changes in $\beta$ and $\alpha$-decay rate of radioactive elements, motion of pulsars and anisotropy of cosmic rays", Am. J. Mod. Phys., 2(3), pp. 177-184, 2013.

[8] Yu. A. Baurov, V.A. Yakovenko, A. V. Komissarov, V.G. Verzhikovski, A.Yu. Baurov, A.A. Konradov, A.A., "Preliminary results of an experimental investigation of a new information channel in nature with the aid of quartz resonators's system", Int. J. Sci. Res. - IJSRU, 16, pp. 469473.Special Issue: Proceedings of the The first UAE International Conference on biological and medical physics, Al-Ain, United Arab Emirates, March 27-30, 2006.

[9] Yu. A. Baurov, "Byuons, Quantum Information Channel, Consciousness and Universe", Open J. Microphys., 1, pp. 3539, 2011.

[10] Yu. A. Baurov, L. Albanese, F. Meneguzzo, V.A. Menshikov, "Universal propulsion harnessing the global anisotropy of the physical space", Am. J. Mod. Phys., 2(6), pp. 383-391, 2013.

[11] Yu. A. Baurov, I.F. Malov, "Variations of Decay Rates of Radio-active Elements and their Connections with Global Anisotropy of Physical Space", Int. J. Pure Appl. Phys., 6(4), pp. 469-482, 2010; Also at: http://arxiv.org/abs/1001.5383.

[12] Yu. A. Baurov, I. F. Malov, I. F., "On the Nature of Dark
Matter and Dark Energy", J. Mod. Phys., 1(1), pp. 17-32, 2010.

[13] Yu. A. Baurov, "The anisotropic phenomenon in the $\beta$ decay of radioactive elements and in other processes in nature", Bull. Russ. Acad. Sci. Phys., 76(10), pp. 1076-1080, 2012.

[14] Yu. A. Baurov, A. Yu. Baurov, A. Yu. Baurov (J), F. Meneguzzo, A. A. Bugaev, "New Interaction in Nature and its Use in the Form of Traction", Int. J. Pure Appl. Sci. Technol., 13(1), pp. 40-49, 2012.

[15] Yu. A. Baurov, Experimental studies of the thrust of an engine -generator model, using the new assumed force in the form of traction, in: A.S. Koroteyev (Ed.), Mater. Sci. Technol. Conf. Relev. Issues Planet. Mission., Russian Academy of Sciences, Moscow, pp. 110-114 (in Russian), 2006.

[16] L. Garrigues, P. Coche, "Electric propulsion: comparisons between different concepts", Plasma Phys. Control. Fusion., 53(12), 124011, 2011.

[17] M. Dudeck, F. Doveil, N. Arcis, S. Zurbach, "Plasma propulsion for geostationary satellites for telecommunication and interplanetary missions", IOP Conf. Ser. Mater. Sci. Eng., 29, 012010, 2012.

[18] A.S. Koroteyev, (ed.), Manned Mission to Mars, Russian Academy of Cosmonautics named after K.E. Tsiolkovsky, Moscow-Korolev, Russian Federation, 2006.

[19] F. Meneguzzo, F., Test of the new concept space thruster, http://youtu.be/F4BWoxMKfNk (movie, last Accessed December 13, 2013).

[20] Yu.A. Baurov, A. Yu. Baurov, Method for flying in outer space, patent Nr. 2338669, Russian Federation, 2007.

[21] Yu. A. Baurov, V.A. Iakovenko, A. V. Komissarov, V.G Verzhikovskiı̌, A.A. Konradov, "Study with a quartz resonator of a new natural informational channel associated with quantum properties of the physical space (vacuum)", Biofizika., 46, pp. 823-828, 2001.

[22] Yu. A. Baurov, A. Yu. Baurov, A. Yu. Baurov (J), R.O. Repchenkov, Method for creating traction force, device for its implementation and means for moving, patent Nr. 2448023, Russian Federation, 2010.

[23] K.A. Trukhanov, Yu. A. Baurov, Cosmophysical Factors and Human Safety in Outer Space, in: Int. Conf. Life Support Syst. as a Means Outer Sp. Explor. by Man, Scientific Council of the RAS for Physiological Sciences -State Research Center of the RF - Institute for Biomedical Problems of the RAS, Moscow, Russian Federation, 2008.

[24] Yu. A. Baurov, A.A. Konradov, V. F. Kushniruk, E.A. Kuznetsov, Yu.G. Sobolev, Yu. V. Ryabov, et al., "Experimental investigations of changes in beta-decay rate of ${ }^{60} \mathrm{Co}$ and ${ }^{137} \mathrm{Cs} "$, Mod. Phys. Lett. A., 16(32), pp. 2089-2101, 2001.

[25] A.G. Parkhomov, "Bursts of Count Rate of Beta-Radioactive Sources during Long-Term Measurements", Int. J. Pure Appl. Phys., 1(2), pp. 119-128, 2005.

[26] Yu. A. Baurov, L. Albanese, F. Meneguzzo, V.A. Menshikov. "Protecting the planet from the asteroid hazard". Int. J. Pure Appl. Phys. Vol. 9, No. 3. pp. 151-168, 2013. 